\title{
Neutrophil Trafficking in Pulmonary Inflammation: Monitoring Migration and Blockade with ${ }^{111}$ In-Labeled Leukocytes
}

Christopher J. Palestro ${ }^{1,2, \#}$, Helena M. Linge ${ }^{3, \#}$, Kenneth J. Nichols ${ }^{1,2}$, Kanta Ochani ${ }^{3}$, Kuldeep K. Bhargava ${ }^{1,2 \#}$ and Edmund J. Miller ${ }^{3,4^{\star} \#}$

${ }^{1}$ Division of Nuclear Medicine and Molecular Imaging, North Shore Long Island Jewish Health System, New Hyde Park, NY, USA

${ }^{2}$ Department of Radiology, Hofstra North Shore-LIJ School of Medicine, Hempstead, NY, USA

${ }^{3}$ Center for Heart and Lung Research, The Feinstein Institute of Medical Research, Manhasset, NY, USA

${ }^{4}$ Departments of Medicine, Molecular Medicine and Surgery, Hofstra North Shore-LIJ School of Medicine, Hempstead, NY, USA

\#Contributed equally to the study

\begin{abstract}
Aim: Although imaging of In vitro labeled leukocytes is commonly used for diagnosing inflammation and infection, data concerning the use of this technique to monitor neutrophil trafficking are scarce. Here we investigated the potential of ${ }^{111}$ In-in vitro labeled leukocytes (InWBC) to monitor neutrophil trafficking in an animal model of pulmonary inflammation.

Methods: F344 rats were divided into 3 groups: Controls (received only InWBC), Inflammation (intra-tracheally challenged with lipoteichoic acid (LTA)+peptidoglycan (PGN) two hours before InWBC injection), and Blockade (pulmonary challenge with receptor blockade [LTAPGN+Antileukinate, a CXC receptor 1 and 2 antagonist]). Leukocytes were obtained from donor rats and labeled with ${ }^{111} \mathrm{In}$-oxine using standard procedures. Labeling efficiency and leukocyte integrity were determined. Animals were administered 3.7-4.6 MBq $\operatorname{lnWBC}$ via the tail vein, and were imaged 18-30 hours later and then euthanized. Post mortem the lungs were lavaged and total and differential alveolar cell counts performed. Lung tissue myeloperoxidase (MPO) activity was determined. Lung, heart, liver, spleen, kidney, marrow, intestine, blood, and muscle were harvested and organ activity/gm tissue determined.
\end{abstract}

Results: InWBC labeling efficiency and cell integrity were not significantly different among groups. InWBC pulmonary activity was significantly higher $(p<0.0001)$ in the Inflammation group $(17.10 \pm 2.04 \%)$ than in the Controls $(1.76 \pm 0.60 \%)$ and the Blockade group $(9.74 \pm 1.14 \%)$. Hemocytometer assessment of the bronchoalveolar lavage fluid revealed that the total number of neutrophils was significantly higher in the Inflammation group than in Controls and the Blockade group. Pulmonary MPO activity $(\mathrm{pg} / \mathrm{mg}$ tissue) was significantly higher $(p<0.01)$ in the Inflammation group $(14.11 \pm 5.56 \%)$ than in Controls $(5.22 \pm 4.77 \%)$ and the Blockade group $(3.66 \pm 3.77 \%)$. InWBC splenic activity was significantly higher $(p<0.0001)$ in the Controls than in the Inflammation and Blockade groups. In the remaining organs, InWBC activity was significantly higher in the Blockade group than in the Control and the Inflammation groups.

Conclusions: In a rat model of pulmonary inflammation using Antileukinate to block neutrophil chemokine receptors, InWBC accurately characterized both pulmonary and extrapulmonary neutrophil trafficking. These data indicate that InWBC may be useful to monitor both pulmonary and extrapulmonary neutrophil trafficking associated with lung inflammation and its regulation.

Keywords: Indium-111; Leukocytes; Neutrophil trafficking; Pulmonary inflammation; Chemokine receptors

\section{Introduction}

In vitro labeled leukocyte imaging is the radionuclide gold standard for imaging most infections in the immunocompetent population [1]. Although there are extensive ( $>30$ years) data demonstrating the role of labeled leukocyte imaging for diagnosing inflammation and infection, data concerning the use of the technique for monitoring leukocyte trafficking are scarce [2-5]. Most of these investigations evaluated time activity curves, and compared the results to known inflammation models, without correlative biochemical/histopathological evaluations. In particular, there are few data on the use of labeled leukocytes to monitor the effects of agents used for neutrophil blockade. The objective of this investigation was to determine the potential of ${ }^{111} \mathrm{In}$-labeled leukocytes (InWBC) to monitor leukocyte trafficking, by comparing the radionuclide results with cellular content of bronchoalveolar lavage and myeloperoxidase (MPO) activity levels, for monitoring neutrophil trafficking in an animal model of pulmonary inflammation with and without neutrophil blockade.

\section{Materials and Methods}

\section{WBC labeling, labeling efficiency and cell integrity determination}

All studies were approved by the Institutional Animal Use and Care
Committee at the Feinstein Institute for Medical Research. Thirty-five young male Fisher rats (250 g, approximately 10-12 weeks of age), including 12 for leukocyte harvesting and labeling purposes, were used. The remaining animals were divided into three groups: Controls $(\mathrm{n}=8)$, Inflammation $(\mathrm{n}=8)$, and Blockade (inflammation+neutrophil blockade; $(n=7))$.

For each group, 15-20 mL blood was withdrawn from four donor rats via cardiac puncture. Rat erythrocytes are not amenable to gravity sedimentation and therefore red cell separation was accomplished using a lysis buffer ( $\mathrm{pH} 7.4)$, containing ammonium chloride (1.5 M), sodium

*Corresponding author: Edmund J. Miller, Center for Heart and Lung Research, The Feinstein Institute for Medical Research, 350 Community Drive, Manhasset, NY 11030, USA, Tel: (516) 562-3401; E-mail: emiller@ nshs.edu

Received August 19, 2015; Accepted October 23, 2015; Published October 27, 2015

Citation: Palestro CJ, Linge HM, Nichols KJ, Ochani K, Bhargava KK, et al (2015) Neutrophil Trafficking in Pulmonary Inflammation: Monitoring Migration and Blockade with ${ }^{111} \mathrm{In}$-Labeled Leukocytes. J Pulm Respir Med 5: 289 doi:10.4172/2161-105X.1000289

Copyright: (c 2015 Palestro CJ, et al. This is an open-access article distributed under the terms of the Creative Commons Attribution License, which permits unrestricted use, distribution, and reproduction in any medium, provided the original author and source are credited. 
bicarbonate $(0.1 \mathrm{M})$, and $1.85 \mathrm{~g}$ disodium EDTA $(0.01 \mathrm{M})$ in sterile water. The $\mathrm{pH}$ was adjusted to 7.4 and the buffer filter-sterilized through a $0.22 \mu \mathrm{m}$ membrane. Red cell lysis was performed by incubating whole blood in a mixture of 1 part lysing buffer and 9 parts sterile water at ambient temperature for ten minutes. After cell lysis and centrifugation (450 g, 10 minutes), the resulting WBC pellet was washed twice in sterile $0.9 \% \mathrm{NaCl}$, resuspended in $0.9 \% \mathrm{NaCl}$, and incubated with $37-$ $41 \mathrm{MBq}{ }^{111} \mathrm{In}$-oxine for 30 minutes. Following repeat centrifugation, 3.7-4.6 MBq InWBC was injected into each anesthetized rat via the tail vein. An aliquot of InWBC was retained for determination of labeling efficiency and cell integrity.

Labeling efficiency (LE) was calculated using the formula $\mathrm{LE}=\mathrm{C} /$ $(\mathrm{C}+\mathrm{W}) \times 100$, where $\mathrm{C}$ was the cell associated activity and $\mathrm{W}$ represented the activity associated with the supernatant. InWBC integrity was assessed by light microscopy using trypan blue exclusion of $\geq 100$ cells [6]. Cell integrity $(V)$ was calculated using the formula $V=N /(N+B)$ $\times 100$, where $\mathrm{N}$ represented the number of intact cells and $\mathrm{B}$ represented the number of labeled leukocytes that had taken up trypan blue as a consequence of lapse in membrane integrity.

\section{Pulmonary inflammation induction}

In the Inflammation and Blockade groups, pulmonary inflammation was induced by the intra-tracheal instillation of the staphylococcal wall components, lipoteichoic acid (LTA) and peptidoglycan (PGN) as previously published [7-9]. Two hours before InWBC injection, animals were anesthetized with an intramuscular injection of ketamine $(45 \mathrm{mg} / \mathrm{kg}$ ) and xylazine $(5 \mathrm{mg} / \mathrm{kg})$. The trachea was surgically exposed and a mixture of LTA $(75 \mu \mathrm{g})$ and PGN $(250 \mu \mathrm{g})$ in $100 \mu \mathrm{l} 0.9 \% \mathrm{NaCl}$ was instilled intratracheally using a 29 -gauge needle. The wound was closed using 5.0 silk sutures and the animals were allowed to recover.

\section{Neutrophil blockade}

In the Blockade group, immediately prior to the intra-tracheal instillation of LTA-PGN, blockade of CXCR1 and CXCR2 on the endogenous neutrophil population was achieved with Antileukinate, a synthetic hexapeptide with acetylated amino- and amidated carboxyterminus (Ac-RRWWCR-NH $\mathrm{N}_{2}$ ). Our previous studies have shown Antileukinate to be a potent inhibitor of CXC chemokine binding to the CXCR1 and CXCR2 receptors, thereby inhibiting neutrophil chemotaxis and degranulation [10-14]. Briefly, the femoral vein was isolated; a 24-gauge cannula was inserted and tied in position. Antileukinate $(10 \mathrm{mg} / \mathrm{kg}$ in $200 \mu \mathrm{l}$ of $0.9 \% \mathrm{NaCl}$ per rat; Syn-Pep Corp, Dublin, CA) was injected followed by $100 \mu \mathrm{L} 0.9 \% \mathrm{NaCl}$. After cannula removal, the vein and skin were closed by sutures. For receptor blockade of the exogenous InWBC, the labeled cells were incubated with $6 \mathrm{mg}$ Antileukinate in vitro for 20 minutes at ambient temperature just prior to tail vein injection.

\section{Ante mortem imaging, euthanasia, bronchoalveolar lavage and organ collection}

Ante mortem imaging was performed in anesthetized rats 18-30 hours after InWBC injection. Ten-minute $128 \times 128$ matrix dorsal images were obtained using a pinhole collimator with a 5-mm-aperture. Energy discrimination was accomplished using 15\% energy windows centered on the $174 \mathrm{keV}$ and $247 \mathrm{keV}$ photopeaks of ${ }^{111}$ In (Figure 1) a five-minute image of the injection site, using the same acquisition parameters, was obtained for quality control purposes.

Immediately after imaging, the anesthetized animals were euthanized by exsanguination via cardiac puncture. Post mortem, the lungs were lavaged twice via the trachea with $7 \mathrm{~mL}$ of $0.9 \% \mathrm{NaCl}$ and

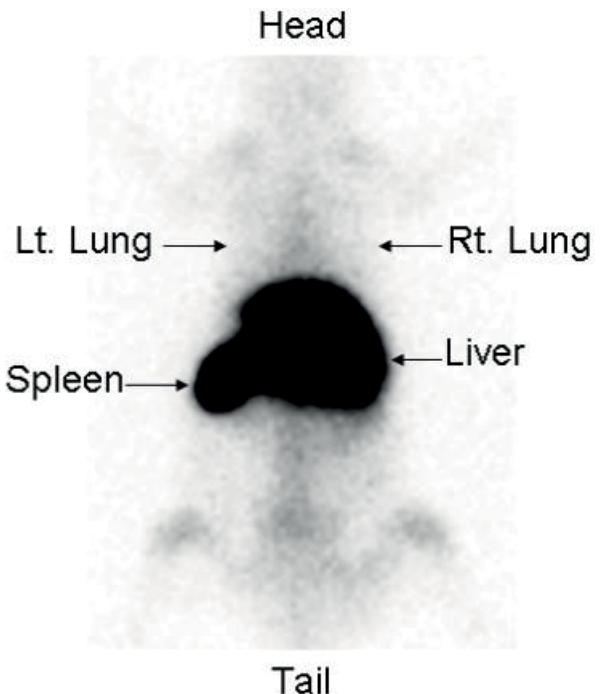

Figure 1: Ten minute dorsal images, using a pinhole collimator, were obtained 18-30 hours after injection of ${ }^{111}$ In-labeled leukocytes.

the resulting bronchoalveloar lavage fluid (BAL) was kept on ice. After centrifugation, the cell pellet was resuspended in Hank's balanced salt solution (HBSS; Gibco, Grand Island NY). Total and differential cell counts were performed by light microscopy using a hemocytometer and assessment of hematoxylin and eosin stained cytospin preparations, respectively. The total number of cells as well as the absolute number and percent of neutrophils recovered from each rat were calculated and recorded. Lung, heart, liver, spleen, kidney, marrow, intestine, blood, and muscle were harvested and ${ }^{111}$ In activity per gram of tissue was determined with a gamma counter (Packard Cobra ${ }^{\circ}$, GMI Inc., Ramsey, $\mathrm{MN})$. Results were expressed as a percent of total tissue activity.

\section{Myeloperoxidase activity assay}

Pulmonary tissue was homogenized using a tissue homogenizer (Tissuemiser, Fisher Scientific, Pittsburg, PA) in 10 volumes (v/w) of lysis buffer (50 mM Tris- $\mathrm{HCl}$ pH7.4; $300 \mathrm{mM} \mathrm{NaCl} ; 2$ mM EDTA; $1 \%$ NP-40, $0.5 \%$ deoxycholic acid, $0.2 \%$ sodium dodecyl sulphate, $1 \mathrm{mM}$ PMSF) supplemented with complete proteinase inhibitor (Roche, Indianapolis, IN). After centrifugation, the supernatant was diluted 7.5-fold in PBS. and assayed for MPO activity in a 96-well plate [15]. All samples were cleared by a dilution step (1:1) in dimethyl-sulphoxide and absorbance measured at $450 \mathrm{~nm}$. MPO activity was calculated from a standard curve using horseradish peroxidase (Pierce, Rockford, IL).

\section{Statistical analysis}

Statistical analysis was performed using commercially available software. Values are reported as mean \pm standard deviation. ANOVA determined whether there were any significant differences among the 3 groups for each organ. Continuous variables were tested by the D'Agastino-Pearson test to determine if they were normally distributed. The paired t-test was used in comparing values between groups for continuous variables that were normally distributed. Frequencies and percentages were used to characterize categorical variables. $\chi^{2}$ analysis of proportions was used to compared ratios. A probability, $\mathrm{p}<0.05$ was considered statistically significant. 
Citation: Palestro CJ, Linge HM, Nichols KJ, Ochani K, Bhargava KK, et al. (2015) Neutrophil Trafficking in Pulmonary Inflammation: Monitoring Migration and Blockade with ${ }^{111}$ In-Labeled Leukocytes. J Pulm Respir Med 5: 289. doi:10.4172/2161-105X.1000289

\section{Results}

Labeling efficiency and cell integrity were similar among all three groups (Table 1). Pulmonaryactivity was significantly higher in the Inflammation group than in the Controls $(\mathrm{p}<0.0001)$ (Table 2,Figure 2). However, receptor blockade of endogenous and exogenous leukocytes resulted in asignificant decrease in pulmonary activity in the Blockade group compared to the Inflammation group $(\mathrm{p}<0.0001) . \mathrm{BAL}$ analysis revealed that the total number of leukocytes in the lungs was significantly higher in the Inflammation group than in the Controls. However, the total number of leukocytes in the Blockade group was not significantly different from Controls (Figure 3). The total number of neutrophils in the Inflammation group was significantly higher than in the Controls and the Blockade group. The total number of neutrophils in the Blockade group was not significantly higher than that in the Controls (Figure 4). The percentage of neutrophils in the Controls was $2.2 \pm 3.4 \%$. This increased significantly to $78.2 \pm 17.3 \%$ in the animals treated with $\mathrm{LTA}+\mathrm{PGN}$ alone. This percentage was significantly decreased in the lavage fluid from the animals of the Blockade group $(13.3 \pm 10.3 \%)$, which was not significantly different from Controls (Figure 5).

Pulmonary MPO activity was significantly higher $(\mathrm{p}<0.01)$ in the Inflammation Group than in the Controls and the Blockade group. There was no significant difference in pulmonary MPO activity between the Controls and the Blockade group ( $\mathrm{p}=0.49)$ (Table 3 ).

Differences in InWBC activity among the three groups were also

Table 1: Labeling efficiency and cell integrity.

\begin{tabular}{|l|l|l|l|}
\hline & Controls & Inflammation & Blockade \\
\hline Labeling Efficiency & $88.80 \%$ & $92.90 \%$ & $88.30 \%$ \\
\hline Cell Integrity & $98.20 \%$ & $98.20 \%$ & $98.5 \%$ \\
\hline
\end{tabular}

The activity in the lung tissue of the Controls was significantly different from that in either the Inflammation or the Blockade groups $p<0.0001$. Additionally, the Blockade group had significantly lower activity than that found in the Inflammation group of animals $p<0.0001$

Table 2: Percent activity/gram lung tissue.

\begin{tabular}{|l|l|l|}
\hline Controls & Inflammation & Blockade \\
\hline $1.76 \pm 0.60 \%$ & $17.10 \pm 2.04 \%$ & $9.74 \pm 1.14 \%$ \\
\hline
\end{tabular}
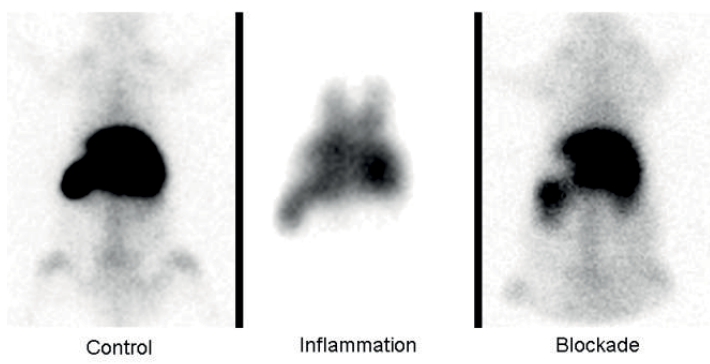

Figure 2: In the Controls, liver, spleen and bone marrow activity are well delineated. There is no discernible pulmonary activity. In the Inflammation group, there is intense, diffuse, bilateral pulmonary activity. In comparison to the Controls there is less activity in the liver and spleen, and no obvious bone marrow activity. Pulmonary activity in the Blockade group, though greater than that in the Controls, is less than the pulmonary activity in the Inflammation group.

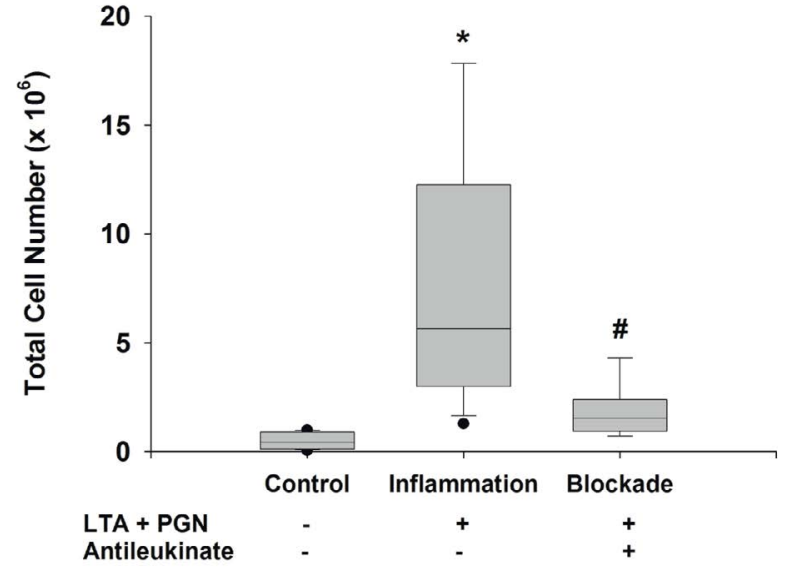

Figure 3: The total number of leukocytes in the lungs was higher in the Inflammation group than in the Blockade group and significantly higher than in Controls $\left({ }^{*} \mathrm{p}<0.05\right)$. The total number of leukocytes in the lungs was not significantly different in the Blockade group than in the Controls.

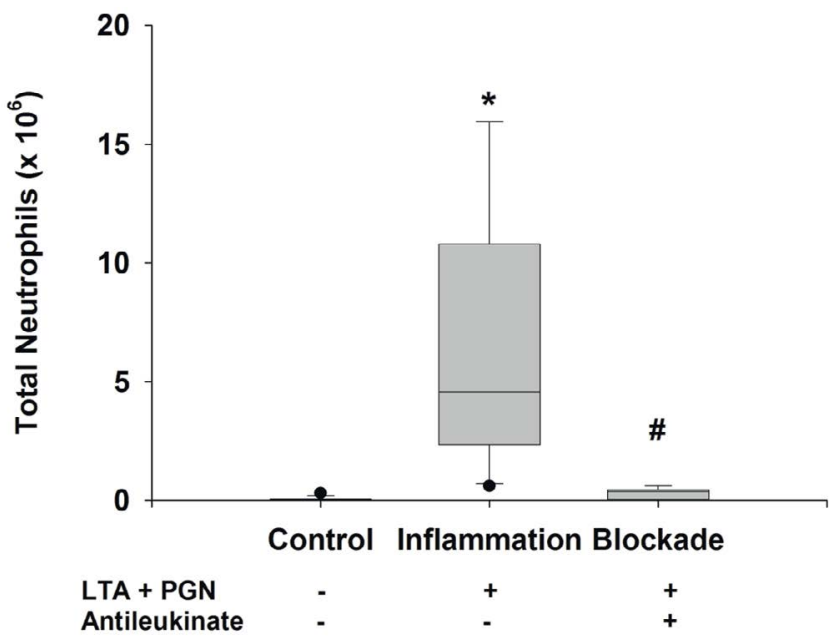

Figure 4: The total number of neutrophils in the Inflammation group was significantly higher than that in the Control and Blockade groups $\left({ }^{*} p<0.05\right)$ The total number of neutrophils in the Blockade group also was not significantly higher than in the Controls (\# >0.05).

observed in organs other than the lungs. There was significantly less InWBC activity in the spleen in the Inflammation and Blockade groups than in the Controls $(\mathrm{p}<0.0001)$. There was significantly more activity in the other organs in the Blockade group than in the Controls and the Inflammation group (Table 4).

\section{Discussion}

Although there are extensive data on the role of InWBC imaging for diagnosing inflammation and infection, there are few data regarding the use of this technique for monitoring leukocyte trafficking. Furthermore, previous investigations focused on stimulation of leukocyte migration rather than its blockade [2-5]. Lewis et al. [5] compared pulmonary migration of radiolabeled neutrophils and eosinophils in guinea pigs challenged with ovalbumin or saline inhalation. They found that in animals challenged five hours before labeled leukocyte injection, there 


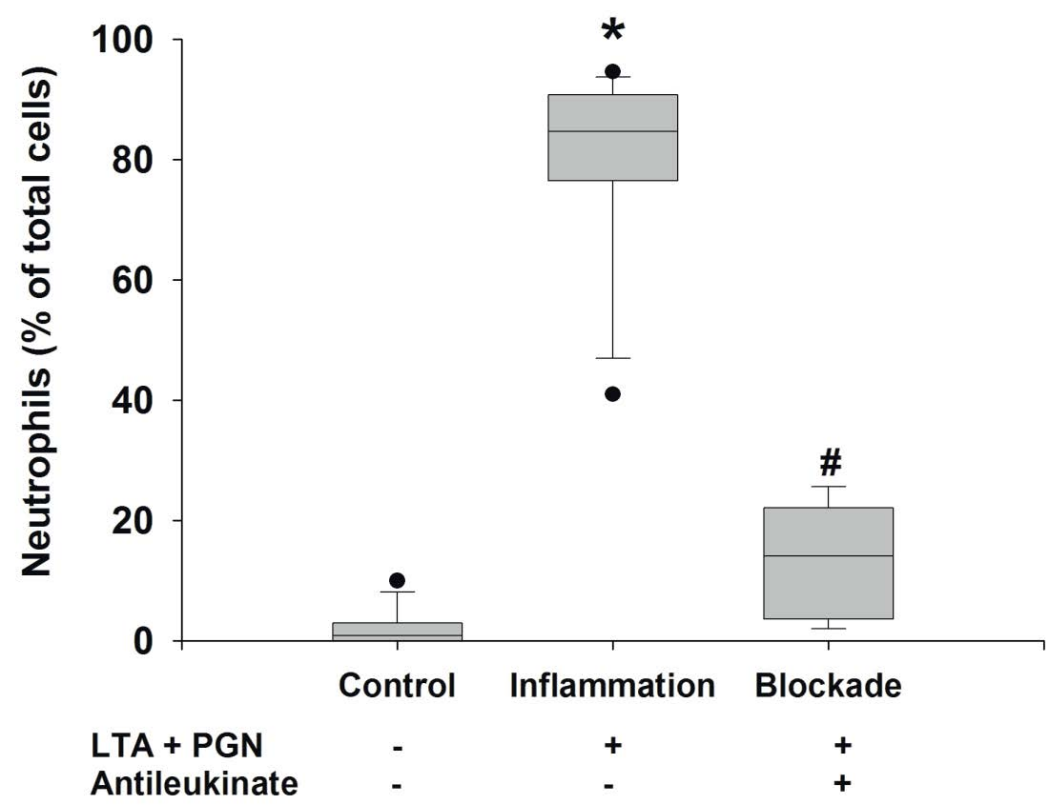

Figure 5: The percentage of neutrophils in the BAL was significantly higher in the Inflammation group than in both the Control and Blockade groups $\left({ }^{*} p<0.05\right)$. There was no significant difference between the Blockade and Control groups $(\# p>0.05)$.

Table 3: Pulmonary MPO levels (pg/mg tissue).

\begin{tabular}{|l|l|l|}
\hline Controls & Inflammation & Blockade \\
\hline $5.22 \pm 4.77$ & $14.11 \pm 5.56$ & $3.66 \pm 3.77$ \\
\hline
\end{tabular}

*\% of total organ activity.

Table 4: Biodistribution of total administered radioactivity*.

\begin{tabular}{|l|l|l|l|l|l|l|l|l|}
\hline & Heart & Liver & Spleen & Kidney & Marrow & Intestine & Blood & Muscle \\
\hline Controls & $0.33 \pm 0.10 \%$ & $20.45 \pm 2.38 \%$ & $70.75 \pm 2.91 \%$ & $2.76 \pm 0.74 \%$ & $2.36 \pm 0.50 \%$ & $0.35 \pm 0.12 \%$ & $0.93 \pm 0.23 \%$ & $0.2 \pm 0.80 \%$ \\
\hline Inflammation & $0.45 \pm 0.23 \%$ & $22.11 \pm 2.08 \%$ & $53.46 \pm 1.98 \%$ & $2.74 \pm 1.64 \%$ & $2.19 \pm 0.70 \%$ & $0.49 \pm 0.25 \%$ & $0.19 \pm 0.13 \%$ & $0.18 \pm 0.15 \%$ \\
\hline Blockade & $2.3 \pm 0.32 \%$ & $27.9 \pm 1.51 \%$ & $37.77 \pm 2.01 \%$ & $10.71 \pm 1.14 \%$ & $3.8 \pm 0.87 \%$ & $2.99 \pm 0.68 \%$ & $3.19 \pm 0.74 \%$ & $1.13 \pm 0.75 \%$ \\
\hline
\end{tabular}

was significantly more radioactivity in the BAL specimens of ovalbumin challenged animals than in saline challenged animals. There was no significant difference in activity, however, between the two groups in animals injected with radiolabeled eosinophils. In animals challenged 24 hours before labeled leukocyte injection, there was significantly more radioactivity in the BAL specimens of ovalbumin challenged animals injected with labeled eosinophils, than in saline challenged animals. In contrast, in animals injected with radiolabeled neutrophils, there was no significant difference in activity between the two groups. The results of that study correlated with known leukocyte migration to the lungs of ovalbumin challenged guinea pigs, namely neutrophilia at 5 hours and eosinophilia at 24 hours after challenge. Dormehl et al. [3] studied neutrophil kinetics in normal and septic shock conditions in a baboon model and reported significantly higher pulmonary retention of labeled neutrophils in animals with septic shock than in controls.

In the current study pulmonary inflammation was induced by intratracheal instillation of LTA and PGN [8]. LTA and PGN are surface associated adhesion amphiphiles present in Gram positive bacteria. Released during bacteriolysis, these potent stimulators of Toll-like receptor 2, when injected locally, work in synergy to stimulate release of pro-inflammatory cytokines and chemokines, including neutrophil chemotaxins and other inflammatory mediators, from surrounding cells and synergistically induce neutrophil migration into the lungs. The derived neutrophilic response to the intratracheal challenge is concentration dependent and in the current study we used a dose that induced pulmonary inflammation, in the absence of severe injury $[8,9]$. The magnitude of the inflammatory response varied considerably between individual animals and as we have seen in other studies the variability is associated with the concentration of the challenge [9] , and the age of the animal [16]. To block neutrophil activation, we used a synthetic hexapeptide, Antileukinate. The peptide, which was originally discovered using a peptide library approach [17], inhibits neutrophil chemotaxis , and degranulation [10] in a variety of models [12, 14, 1820]. Importantly, receptor inhibition reduced both the number of cells that migrated to the site of challenge, and the variability of the response following pulmonary challenge.

We found that InWBC results accurately characterized stimulation of neutrophil migration in pulmonary inflammation. InWBC activity was significantly higher $(\mathrm{p}<0.0001)$ in the lungs of the Inflammation group, than in the lungs of the Controls. The radiolabeled leukocyte results, moreover, closely paralleled those of pulmonary MPO activity and, as measured in BAL, the total leukocyte count, total neutrophil count and percent neutrophils in the lungs. Our results are in agreement with the findings reported by Lewis et al. [5] and Dormehl et al. [3].

Our data also indicate that, in addition to monitoring the effects of stimulating agents on neutrophil migration, it may be possible to use InWBC to monitor the effects of blocking agents on neutrophil 
Citation: Palestro CJ, Linge HM, Nichols KJ, Ochani K, Bhargava KK, et al. (2015) Neutrophil Trafficking in Pulmonary Inflammation: Monitoring Migration and Blockade with ${ }^{111}$ In-Labeled Leukocytes. J Pulm Respir Med 5: 289. doi:10.4172/2161-105X.1000289

Page 5 of 5

migration. Pulmonary activity was significantly lower in the Blockade group than in the Inflammation group, as were MPO activity, absolute number of neutrophils, and neutrophils as a percent of total leukocytes in the lungs.

In addition to differences in pulmonary activity among the three groups, we observed differences in other organs as well. In the Controls, InWBC accumulated primarily in the spleen, liver and bone marrow. In the Inflammation group, splenic activity decreased as the labeled leukocytes were redirected to the lung, indicating altered InWBC trafficking. This observation is in agreement with what previously has been reported by Dormehl et al. [3]. In the Blockade group, there was increased activity in all other organs, except the spleen, compared to the Controls and the Inflammation group. We did not however observe any increase in MPO activity in these other organs (data not shown). This likely is due to differences in method sensitivity. The exact reason for the increased organ activity in the Blockade group remains uncertain. One possibility is related to the effect of proinflammatory mediators on the normal neutrophil life span. Circulating neutrophils have always been considered to be short lived, with estimates of half-life in rodents varying from 1.5-12.5 hrs under normal conditions [20-22].

However, there are many factors that may delay normal apoptosis. Exposure to endogenous or exogenous proinflammatory mediators (such as CXCL2 or LTA) and extravasation into the alveolar space prolong neutrophil lifespan $[20,23,24]$. It is possible that this may explain our results. It also is possible that the altered distribution observed in the Blockade group is a direct effect of Antileukinate itself. This, however, was beyond the scope of this investigation.

In summary, in a rat model of pulmonary inflammation using Antileukinate to block neutrophil receptor recognition of major neutrophil chemotaxins, InWBC accurately characterized neutrophil trafficking to the lungs and potentially may be able to provide data about leukocyte migration and fate under normal conditions as well as the effects of various agents, both stimulants and immunosuppressants (e.g. glucocorticoids), on leukocyte migration in homeostasis and in diseased states. The data also suggest that InWBC may be useful to monitor the effects of lung inflammation and its regulation of extrapulmonary neutrophil trafficking.

\section{Acknowledgements}

The authors thank Ms. Erica Spessot for assistance with editing of the manuscript.

\section{References}

1. Palestro CJ, Love C, Bhargava KK (2009) Labeled leukocyte imaging: current status and future directions. Q J Nucl Med Mol Imaging 53: 105-123.

2. Das DK, Steinberg H, Bandyopadhyay D, Hoory S (1988) Potential use of indium-111-labeled polymorphonuclear leukocytes for the detection of lung microvascular injury. J Nucl Med 29: 657-662.

3. Dormehl IC, Maree M, Cromarty D, Böckmann H, Jacobs L, et al. (1990) Investigation by scintigraphic methods of neutrophil kinetics under normal and septic shock conditions in the experimental baboon model. Eur J Nucl Med 16: 643-647.

4. Lewis CA, Farr SJ, Broadley KJ (1996) Development of a method for monitoring the migration of $111 \mathrm{ln}$-labeled eosinophils and neutrophils to the lungs of anaesthetized guinea pigs by gamma scintigraphy and bronchoalveolar lavage. J Immunol Methods 190: 51-60.

5. Lewis CA, Warren SJ, Broadley KJ (1999) 111In-labelled leukocyte migration to the lungs of ovalbumin-sensitized guinea-pigs after aerosol challenge with ovalbumin monitored by gamma scintigraphy. Int Arch Allergy Immunol 118: 51-58.

6. Miller EJ, MacArthur CK, Gray LD, Cohen AB (1990) Liberation of a neutrophil enzyme-releasing peptide from the surface of human alveolar macrophages.
Am J Physiol 258: L328-333.

7. De Kimpe SJ, Kengatharan M, Thiemermann C, Vane JR (1995) The cell wall components peptidoglycan and lipoteichoic acid from Staphylococcus aureus act in synergy to cause shock and multiple organ failure. Proc Natl Acad Sci U S A 92: 10359-10363.

8. Zhang Y, Lin X, Koga K, Takahashi K, Linge HM, et al. (2011) Strain differences in alveolar neutrophil infiltration and macrophage phenotypes in an acute lung inflammation model. Mol Med 17: 780-789.

9. Linge HM, Lee JY, Ochani K, Koga K, Kohn N, et al. (2015) Age influences inflammatory responses, hemodynamics, and cardiac proteasome activation during acute lung injury. Exp Lung Res 41: 216-227.

10. Hayashi S, Kurdowska A, Miller EJ, Albright ME, Girten BE, et al. (1995) Synthetic hexa- and heptapeptides that inhibit IL-8 from binding to and activating human blood neutrophils. J Immunol 154: 814-824.

11. Hayashi S, Yatsunami J, Fukuno Y, Kawashima M, Miller EJ (2002) Antileukinate, a hexapeptide inhibitor of CXC-chemokine receptor, suppresses bleomycin-induced acute lung injury in mice. Lung 180: 339-348.

12. Hirayama S, Shiraishi T, Shirakusa T, Higuchi T, Miller EJ (2006) Prevention of neutrophil migration ameliorates rat lung allograft rejection. Mol Med 12: 208213

13. Lin X, Yang H, Sakuragi T, Hu M, Mantell LL, et al. (2005) Alpha-chemokine receptor blockade reduces high mobility group box 1 protein-induced lung inflammation and injury and improves survival in sepsis. Am J Physiol Lung Cell Mol Physiol 289: L583-590.

14. Miller EJ, Cohen AB, Peterson BT (1996) Peptide inhibitor of interleukin-8 (IL-8) reduces staphylococcal enterotoxin-A (SEA) induced neutrophil trafficking to the lung. Inflamm Res 45: 393-397.

15. Suzuki K, Ota H, Sasagawa S, Sakatani T, Fujikura T (1983) Assay method for myeloperoxidase in human polymorphonuclear leukocytes. Anal Biochem 132: 345-352.

16. Houghten RA, Pinilla C, Blondelle SE, Appel JR, Dooley CT, et al. (1991) Generation and use of synthetic peptide combinatorial libraries for basic research and drug discovery. Nature 354: 84-86.

17. Lomas-Neira JL, Chung CS, Grutkoski PS, Miller EJ, Ayala A (2004) CXCR2 inhibition suppresses hemorrhage-induced priming for acute lung injury in mice. J Leukoc Biol 76: 58-64.

18. Maus U, von Grote K, Kuziel WA, Mack M, Miller EJ, et al. (2002) The role of $\mathrm{CC}$ chemokine receptor 2 in alveolar monocyte and neutrophil immigration in intact mice. Am J Respir Crit Care Med 166: 268-273.

19. Min JH, Codipilly CN, Nasim S, Miller EJ, Ahmed MN (2012) Synergistic protection against hyperoxia-induced lung injury by neutrophils blockade and EC-SOD overexpression. Respir Res 13: 58

20. Kolaczkowska E, Kubes $P$ (2013) Neutrophil recruitment and function in health and inflammation. Nat Rev Immunol 13: 159-175.

21. Pillay J, den Braber I, Vrisekoop N, Kwast LM, de Boer RJ, et al. (2010) In vivo labeling with $2 \mathrm{H} 2 \mathrm{O}$ reveals a human neutrophil lifespan of 5.4 days. Blood 116 : 625-627.

22. Tak T, Tesselaar K, Pillay J, Borghans JA, Koenderman L (2013) What's your age again? Determination of human neutrophil half-lives revisited. J Leukoc Biol 94: 595-601.

23. Hu M, Miller EJ, Lin X, Simms HH (2004) Transmigration across a lung epithelial monolayer delays apoptosis of polymorphonuclear leukocytes. Surgery 135 87-98

24. Lotz S, Aga E, Wilde I, van Zandbergen G, Hartung T, et al. (2004) Highly purified lipoteichoic acid activates neutrophil granulocytes and delays their spontaneous apoptosis via CD14 and TLR2. J Leukoc Biol 75: 467-477. 